\title{
Smart monitor of pacemaker patient by using iot cloud in real time
}

\author{
Jameel Kadhim Abed, Hassnaa Mustafa Abed
}

Electrical Technical Engineering College, Middle Technical University, Iraq

\begin{abstract}
Article Info
Article history:

Received Jun 16, 2019

Revised Sep 17, 2019

Accepted Oct 8, 2019

\section{Keywords:}

ECG sensor

Heart rate

IoT cloud

Pacemaker patient

Wearablemonitoring

ABSTRACT

People severing from heart diseases are increasing day after day, now it's more important to focus on this destructive problem and provide a good health care and remote patient monitoring. An effective monitoring system has been discovered to monitor patients who are implanting a pacemaker and need long-term follow-up. So extensive research has been done to monitor ECG and analyze it by using the free open source website and linking it with portable ECG system that are responsible for the presentation and storage of data to the doctor and patient. In this research we will develop a new method of ECG monitoring for patients who are implanting pacemakers and help them to transfer their ECG signal for long distances depending on the Internet things (IOT). Once the device is worn, it will start collecting ECG information and sent it directly via Wi-Fi to the IOT cloud. Reviewing ECG data through the web has led to the alleviation of the problem of the inability of the doctor to monitor the patient from the home as well as in the event that the doctor is being in another country, the device also helps to discover the magnetic field that confuses the pacemaker by giving the patient a warning. The results of the test are able to detect that the system would be reliable for displaying real-time ECG data, which could help facilitate patient follow-up after implantation of the pacemaker at home with ease of use and cheaper price.
\end{abstract}

Copyright (ङ 2020 Institute of Advanced Engineering and Science. All rights reserved.

\section{Corresponding Author:}

Jameel Kadhim Abed,

Electrical Technical Engineering College,

Middle Technical University, Baghdad, Iraq.

Email: hassnaamustafa@gmail.com

\section{INTRODUCTION}

Population and medical expenses are increasing, health care has become the issue of the age for every world specialized in the field required, patients' heart pacemaker is increasing and need to follow-up continuous and the lowest possible costs. Because of the large number of patients with pacemakers and the need to follow them continuously, ECG has been applied before implantation and after implantation, the patient is usually associated with 12 electrodes and then combines electrocardiogram which is good in the short term not to enter Hospital for a long period of time, increasing the burden on hospitals. a portable ECG system that helps these patients after implantation of a pacemaker has been discovered and can be returned to his home to follow up with the doctor without going to hospital [1-2].

A fact that the patient with a pacemaker needs to be followed periodically by the doctor after implanting the device. So when the doctor is in another place and can not reach the pataient for some reason or the high cost of hospital for the patient to stay, this device can make a sulotion [3-4].

The paper presents a wireless system after implantation based on the ECG and Internet Objects (IOT). Based on this system, we design a surveillance system that enables the patient to wear and transfer information directly to Internet objects (IOT) using Wi-Fi, compared to other ways such as Bluetooth, Zigbee. Wi-Fi can send more data and provide a cloud (www.Thinger.com) Can be moved anywhere in the 
world. To obtain real-time ECG data without delay for users, this is done by the HTTP server in the Internet Object Cloud (IOT) [5-6], it is free and easy to connect to a Wi-Fi network and in strict confidentiality [6-7]. The system is show in Figure 1.

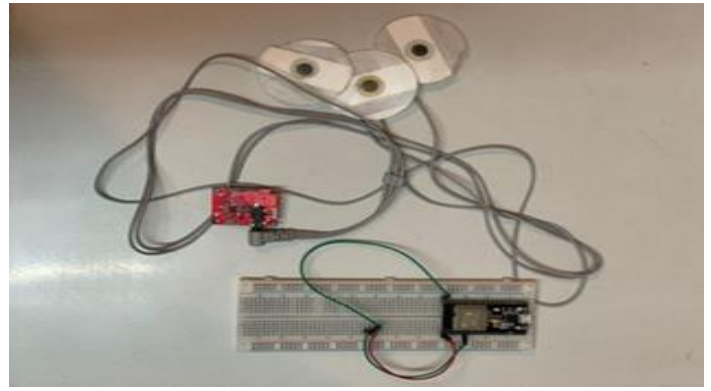

Figure 1. A wireless system with ECG sensor

This paper is arranged for several sections; first section displays the proposed method of IOT and portable monitoring system. Another section is applied to the system with the practical model, which includes Internet cloud and the website (www.Thinger.com) that used to transfer data, in the end a real examination of a patient that puts a pacemaker, in order to see if the doctor has received the required reading in real time.

\section{THE PROPOSED METHOD OF IOT AND PORTABLE MONITORING SYSTEM}

The Construction of the IoT and Portable Monitoring System is show in Figure 2 [8-9]. The starting point of the system that is responsible for collecting data from the surface of the human body and transferring it to the cloud (website) through Wi-Fi. It can record the data for hours that may reach the day. This data is processed through several stages of amplification and filtering, as well as using a specific wireless protocol that consumed a medium energy [10-11].

The interface that is responsible for displaying the data through which a doctor will read the ECG which he will open with any device and thus using the website (www. Thinger.com) is designed to be an accessible tool. It is also free and the user can log into the cloud to obtain real-time visual data. Web pages are more flexible in terms of maintenance and development as well as their ease of presence on the laptop and smart phones as soon as the Internet is available [12-13]

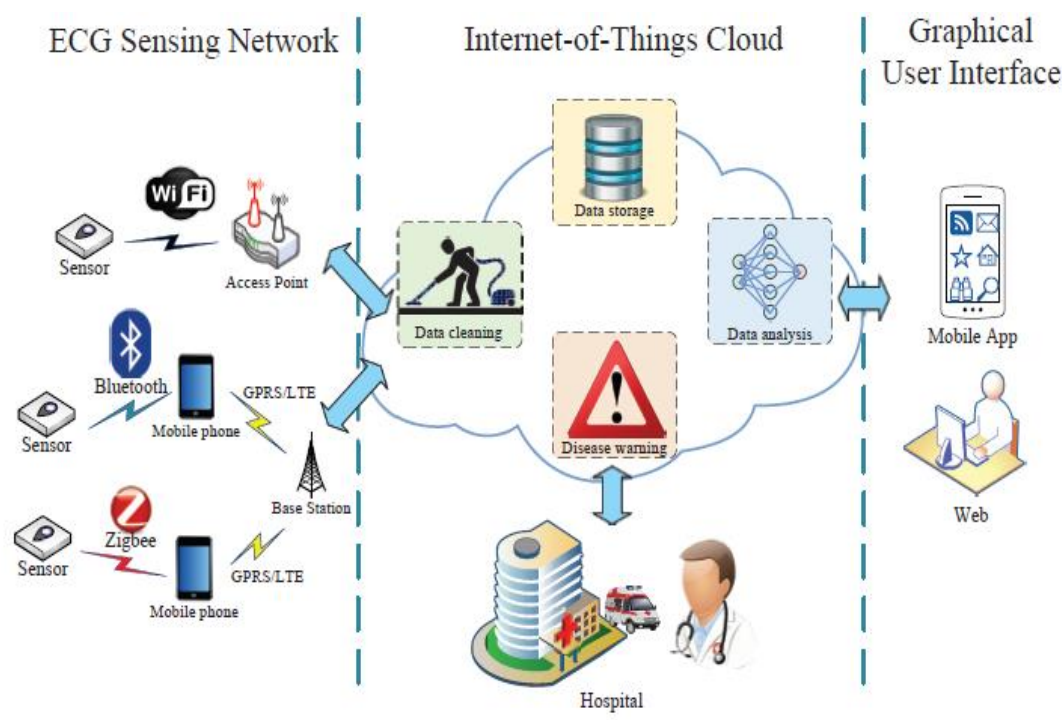

Figure 2. The proposed method IoT and portable monitoring system [5] 
After making the proposed device we connect the device with the site using the method used in the site by installing three points of connection located on the site as shown in the Figure 3 and Figure 4. Also the three points written in the programming code that used in the controller.

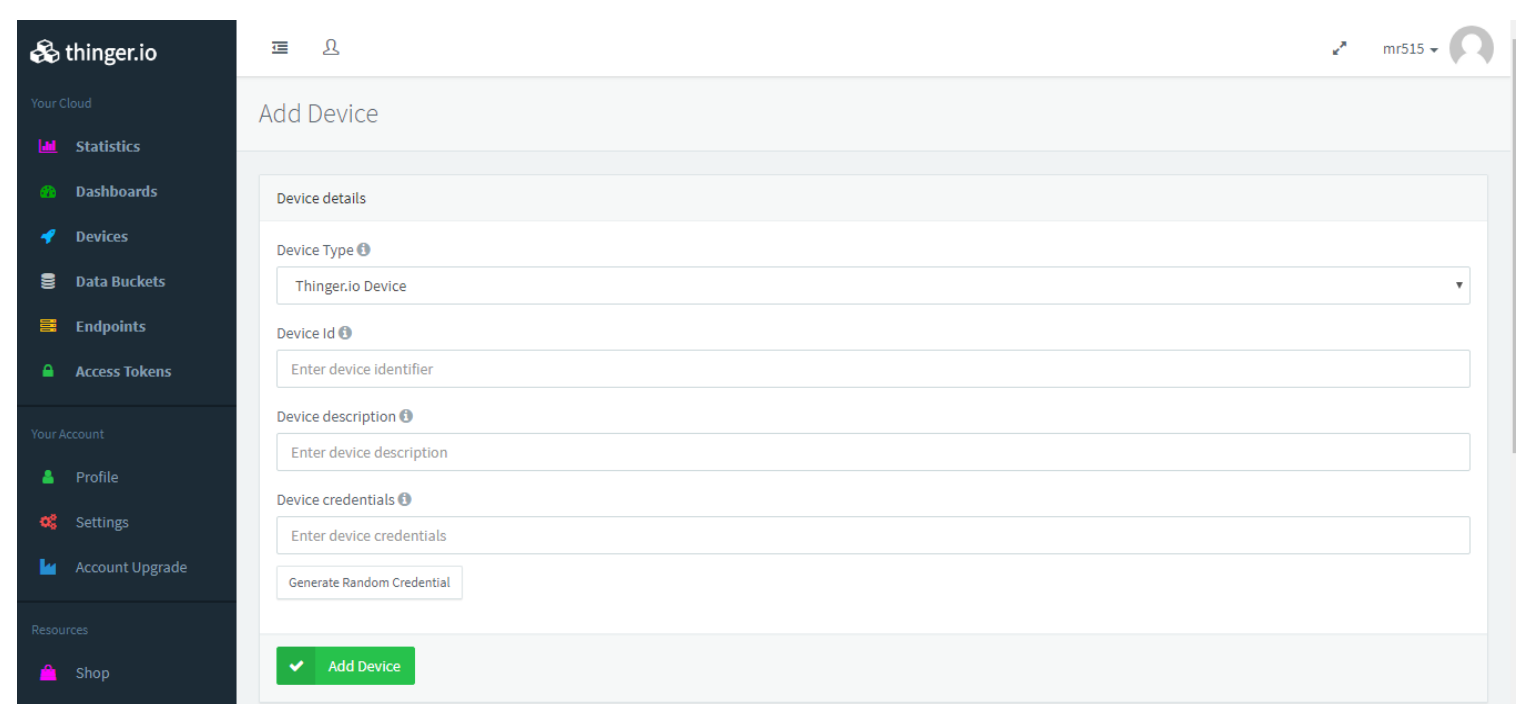

Figure 3. The three points connect the device with controller

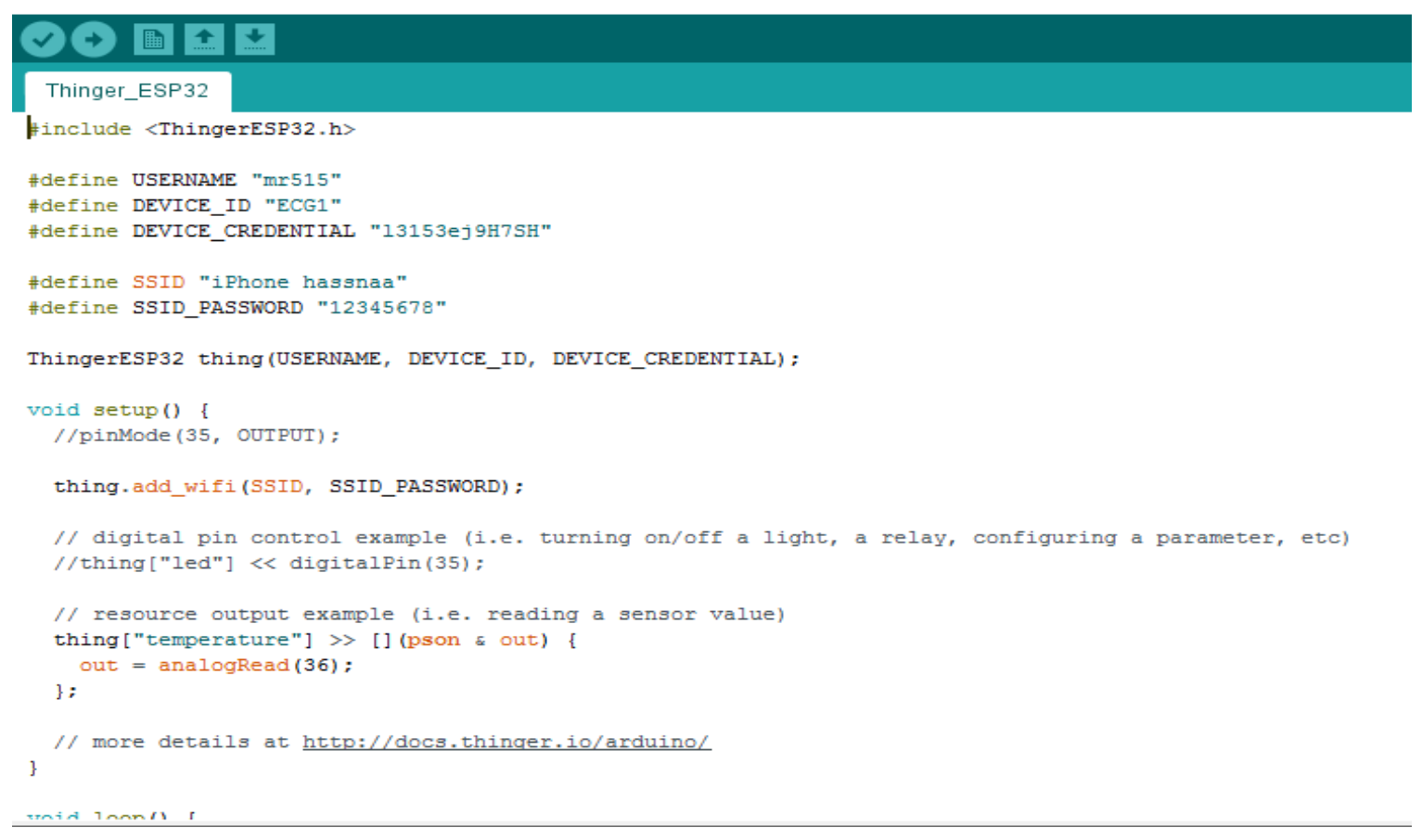

Figure 4. The three points written in the code

\section{MODEL OF THE SYSTEM AND COMPONENTS}

According to the structure written in the second Section 2, monitoring system based on ECG Sensor, cloud computing and the Web. The monitor system shows the ECG node that collects data from the surface of the human body by installing it in a well-known way and then sends it to the Web via Wi-Fi. As shown in Figure 5. 


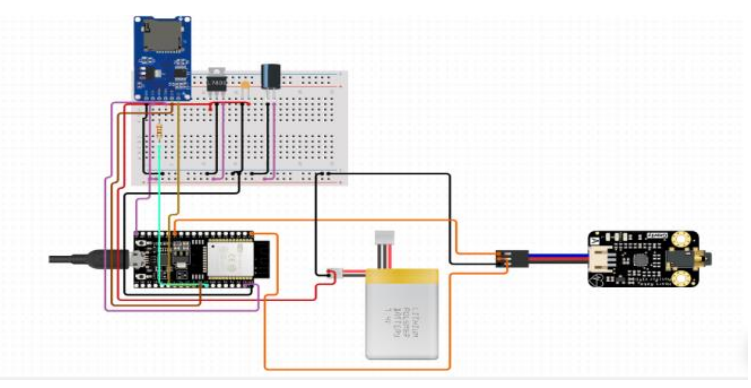

Figure 5. The monitor system

\subsection{Components of the Monitor System}

\subsubsection{ECG Sensor}

With the help of the EC8232 ECG sensor, ECG data is collected from the surface of the body, which consists of dry electrodes the signal passes through the AD8283 band pass filter for remove the unwanted frequency that is outside the calculated frequency range of the signal $(0.5 \mathrm{~Hz}$ and $100 \mathrm{~Hz})$, and then its amplified by the operational amplifier. Finally, ECG were collected are from (0 V to 3.3V) [14-15].

\subsubsection{Controller}

Is the basis that controls the ECG control node, we use a special unit to process the collected ECG signal and send it to a Wi-Fi, module it use the ESP32 chip that designed for Internet-of-Things (IoT) applications and wearable sensors. It has been chosen because it features the modern properties of lowenergy chips, such as a different energy pattern, high efficiency gates around the clock, and power scaling and other features. It has been chosen because it features the modern properties of low-energy chips such as a different energy pattern, high-efficiency gates around the clock, and power scaling and other features. ESP30 is periodically updated and according to the specific situation. For example, a low cycle is used to reduce the amount of energy spent by the chip. The output can be adjusted to the power amplifier, thus helping the development in communications, power consumption and data rate. The main chip consists of four actions, sampling, ADC, storage samples, and packages. At first, the analog ECG signal is numbered and samples are taken and transferred through the ADC. These ECG samples are then stored temporarily in the buffer before filling in the desired format. Finally, the data is transferred to Wi-Fi, This controller has a $2.4 \mathrm{GHz} \mathrm{Wi}-\mathrm{Fi}$ network, a 32-bit dual-core chip with 600 DMIPS, a toolkit, API, components and workflow for applications using Windows, Linux and Mac OS, the Specifications of ESP32 shown in Figure 6 [16-17].

\begin{tabular}{l|l}
\hline \multicolumn{2}{c}{ Specifications } \\
\hline Operating Voltage & $2.2 \mathrm{~V}$ to $3.6 \mathrm{~V}$ \\
\hline GPIO & 36 ports \\
\hline ADC & 14 ports \\
\hline DAC & 2 ports \\
\hline Flash memory & $16 \mathrm{Mbyte}$ \\
\hline SRAM & $250 \mathrm{Kbyte}$ \\
\hline Clock Speed & $\mathrm{Up} \mathrm{to} 240 \mathrm{MHZ}$ \\
\hline Wi-Fi & $2.4 \mathrm{GHz}$ \\
\hline Sleep Current & $2.5 \mu \mathrm{A}$ \\
\hline
\end{tabular}

Figure 6. Specifications of controller (ESP32) [10]

\subsubsection{Website}

Thinger (https://console.thinger.io) the best Platform with scalability, speed, security and easy coding all this to manage your IoT that gives a cloud service designed to allow Receive data from sensor to be showed on the internet and be viewed in real-time and any place in world. This website has a very important feature is that anyone can handle it with the ability to register on the accounts for free to start the Internet of thing (IoT) project in minutes. Easily con Figureure the board by connected between the chip esp32 and the web by define the Username, Device ID and Device CREDENTIAL these written in the code of the chip. As shown in Figure 7 [18]. 


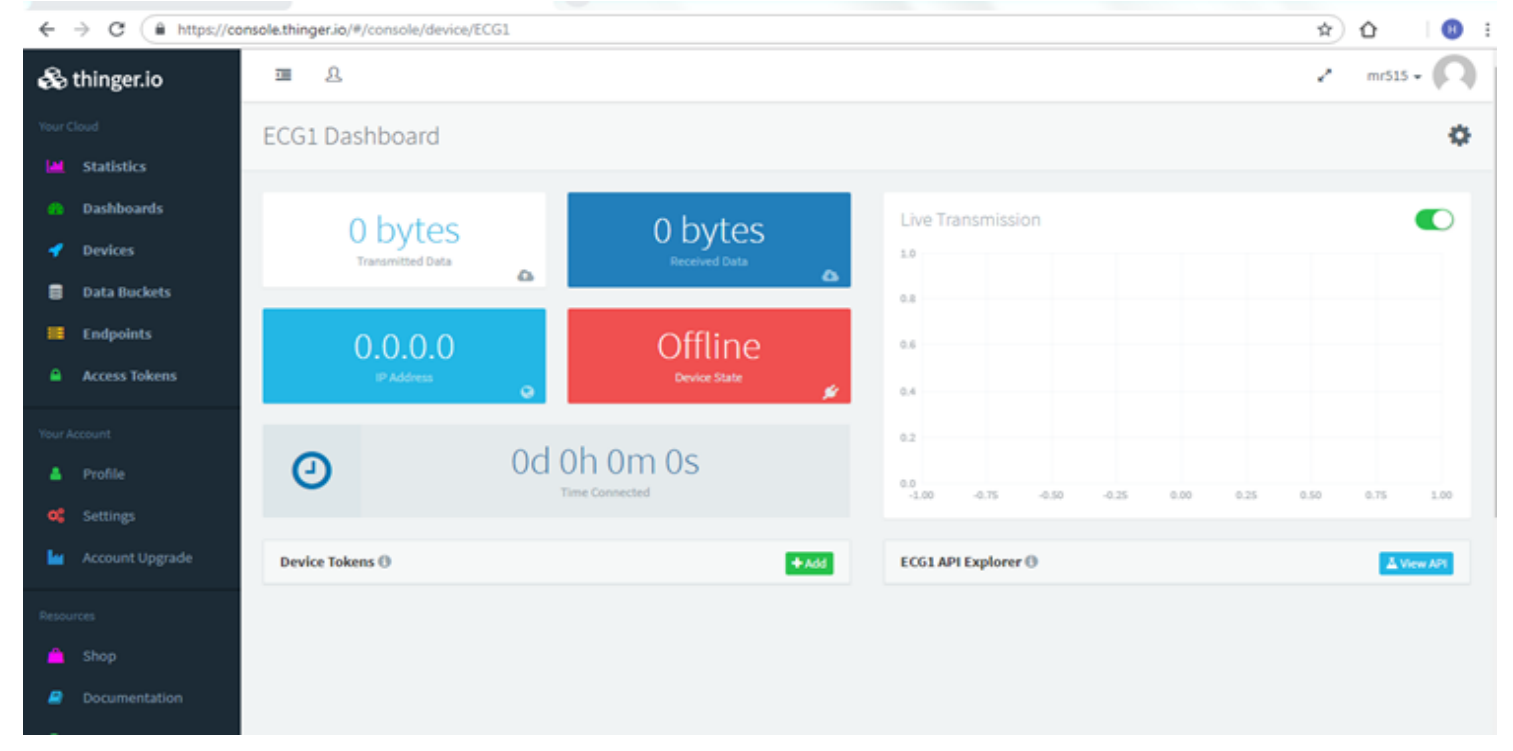

Figure 7. Example of conFigureuration with free register account

\subsubsection{Micro SD Module}

The micro SD card moduleit is a chip that can easy to add data from the sensor after record the data to save it with Microsoft Excel Worksheet to read by a doctor. The operating voltage of any standard micro SD Cards is $3.3 \mathrm{~V}[19]$.

Rechargeable 3.7 V Lithium Batteries

Full Charge Output Voltage: 4.2V - 3.7V with Connector Genuine JST and Includes over-charge, over-use, and short circuit protection [20].

\section{RESULTS AND ANALYSIS}

This section is divided into two subsections: the experiment receiving the signal on the normal patient and the patient who has the pacemaker and the second section the signal analyzer received for the patient who has a pacemaker and calculate the heart rate.

\subsection{The Experiment Receiving the Signal}

Three electrodes--lead placed on the surface of the body of patient body to capture the signal of the ECG In order to get the best single of the ECG, the electrodes must place in a triangle around the heart as shown in in Figure 8 [21].

The single that record from the proposed system is show in Figure 9 is for normal patient and the second one for patient that has pacemaker as showen in Figure 10.
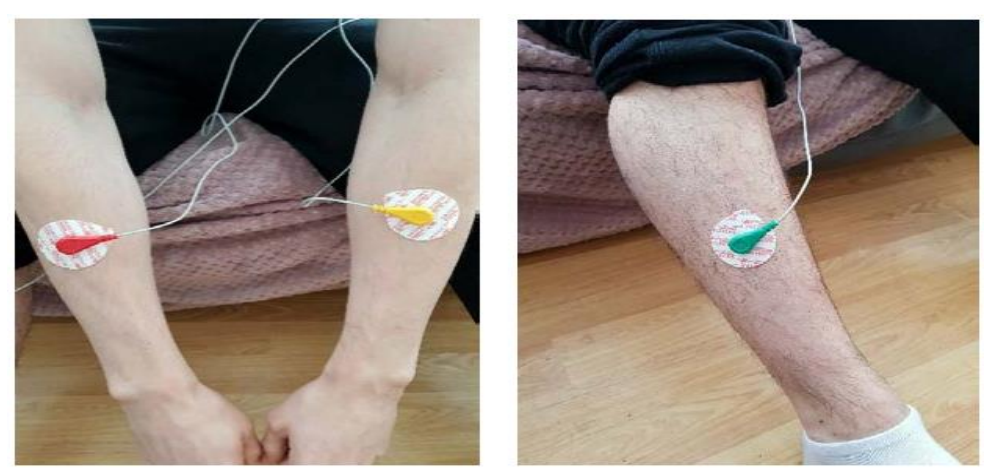

Figure 8. The placement of electrodes 


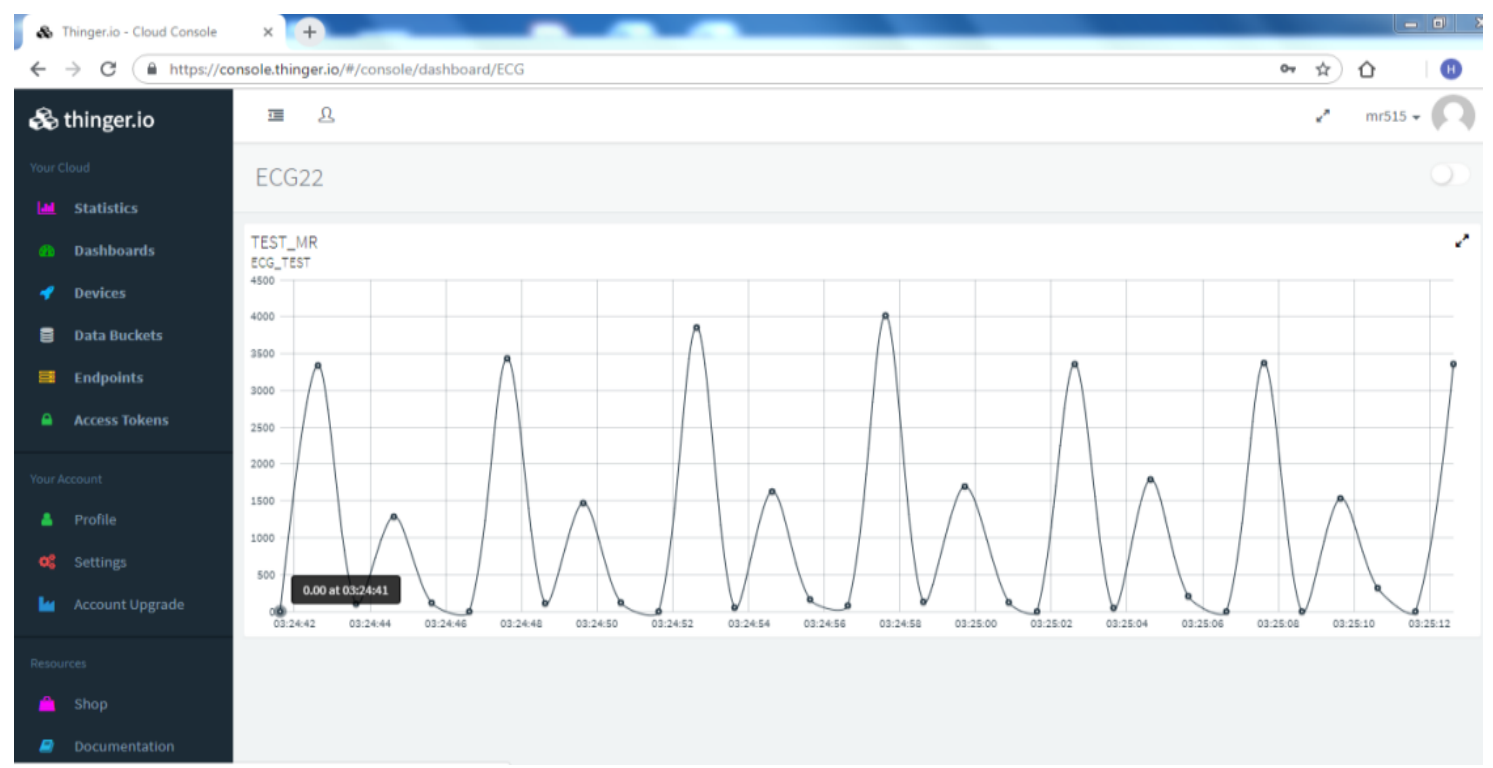

Figure 9. The single for normal patient

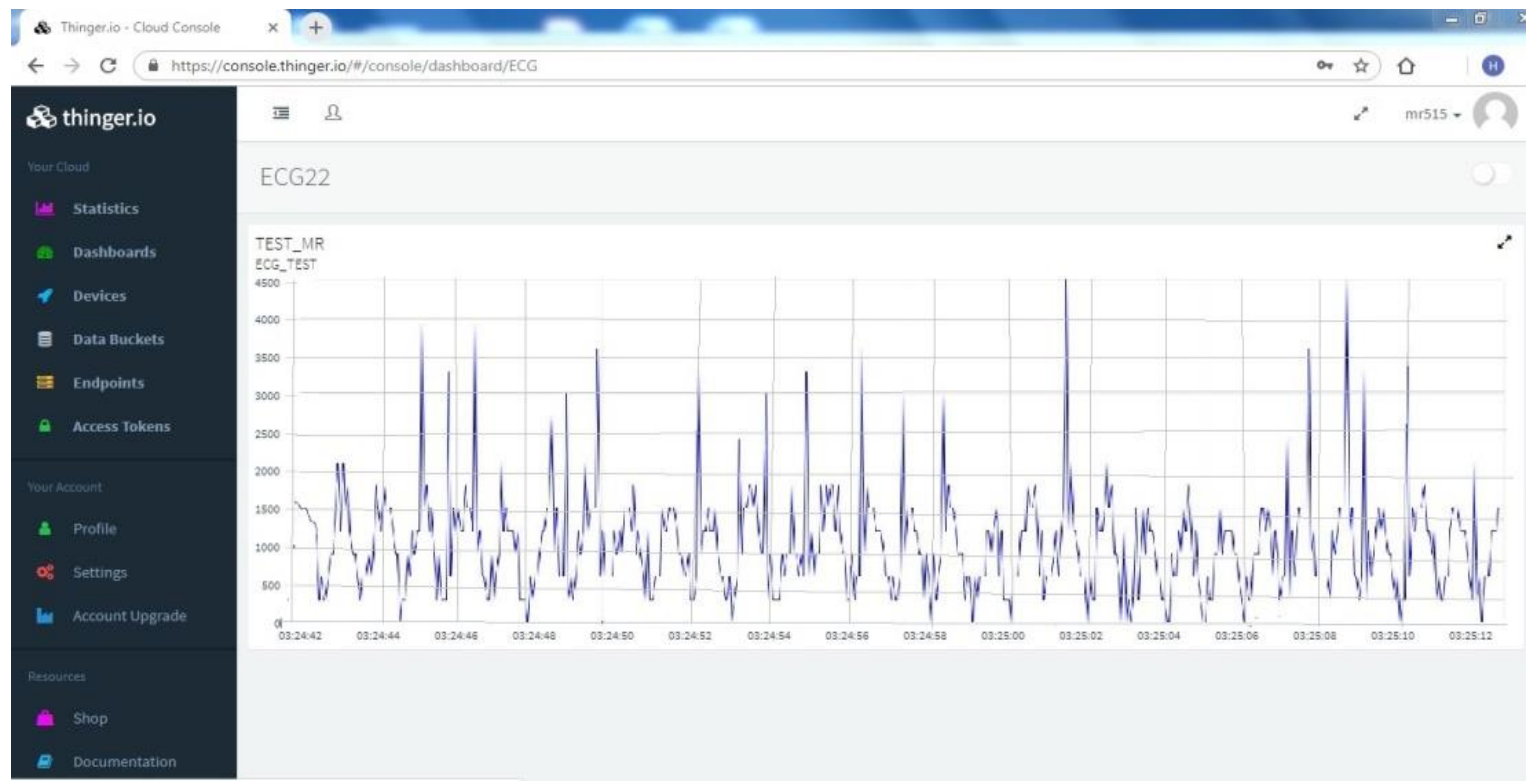

Figure 10. The single for patient has pacemaker

\subsection{Analysis}

\subsubsection{Mathematical Equations of the Theoretical Method}

The interval of these $\mathrm{R}$ waves is usually used to know the heart rate. There is many ways to measure the heart rate through the ECG single. The data collected from the regular volunteer to take the heart rate where the patient in place 1(website in Browser A) and the doctor in place 2 (website in Browser B), the doctor must know if the rhythms is regular or irregular [22-23].

Both cases there are special accounts which are as follows:

Case. 1 (if the rhythm regular)

The distance between each $\mathrm{R}$ wave and anther is equal. We used by calculate the number of big square between R1 and R2 which is divided for five small square:

1 big square $=$ five small square

We have as shown in Figure 11(a)

2 big square and 2 half big square $=2 * 5+2+2=14$ the number of small square 
Then we use the equation:-

Heart rate $=1500 /$ number of small square

$=1500 / 14$

$=107$ in $60 \mathrm{sec}$

Case. 2 (if the rhythm irregular)

The distance between each $\mathrm{R}$ wave and anther is unequal.

We used by calculate the number of $\mathrm{R}$ waves through

15 big squares and multiplying it by 20 like in the Figure 11(b)

Heart rate $=$ number of $\mathrm{R}$ wave in 15 big square* 20

$=5 * 20$

$=100$ in $60 \mathrm{sec}$

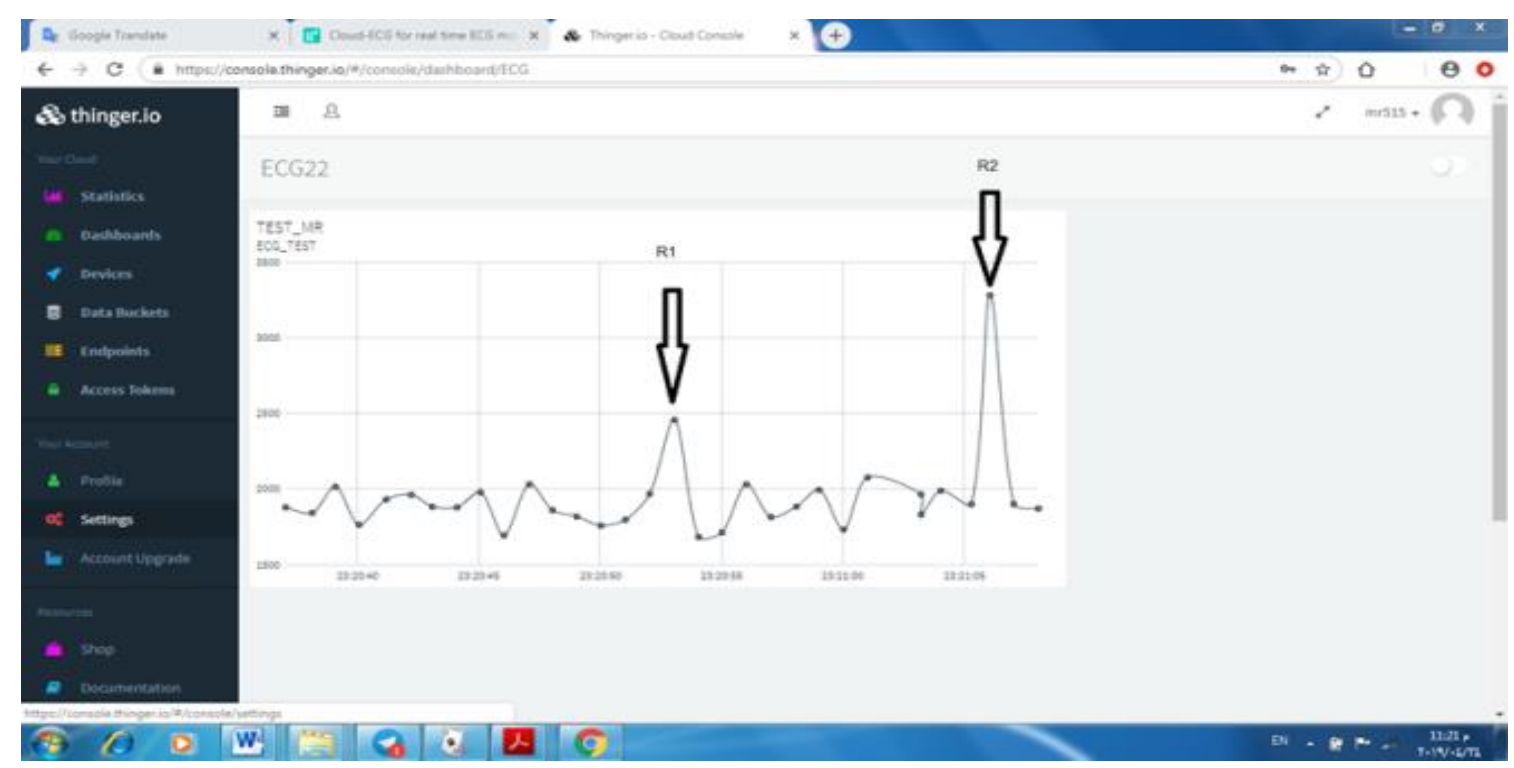

Figure 11. (a) case. 1

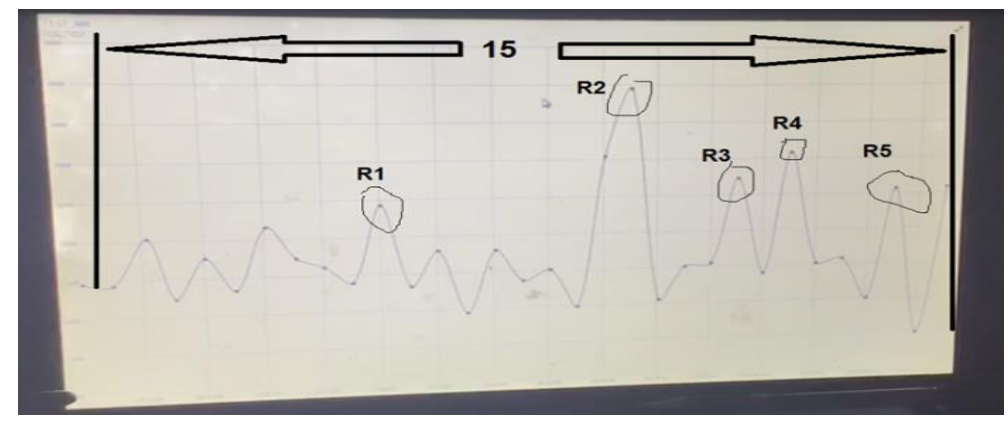

Figure 11. (b) Case. 2

\subsubsection{Practical Method}

The trials were conducted by 10 patients to confirm the measured value of the male and female heart rate between the ages of 20 and 30 years. Their weights were between 60 and 90 and patients had to sleep or sit without moving. An electronic pressure measuring device was used to measure the heart rate and then use the system The proposal for each reading is similar and according to the written table. The summary results, shown in Table 1 and Figure 12 first patient with heart beat [24-26]. 
Table 1. The Result of Heart Rate Measurement Comparing between Proposed System and Digital Blood Pressure

\begin{tabular}{ccc}
\hline Patient Tester & Heart Rate from Digital Blood Pressure Monitor & Heart Rate from proposed system \\
\hline 1 & 69 & 76 \\
2 & 72 & 73 \\
3 & 87 & 81 \\
4 & 93 & 85 \\
5 & 82 & 84 \\
6 & 87 & 80 \\
7 & 69 & 72 \\
8 & 56 & 65 \\
9 & 75 & 71 \\
10 & 80 & 84 \\
\hline
\end{tabular}

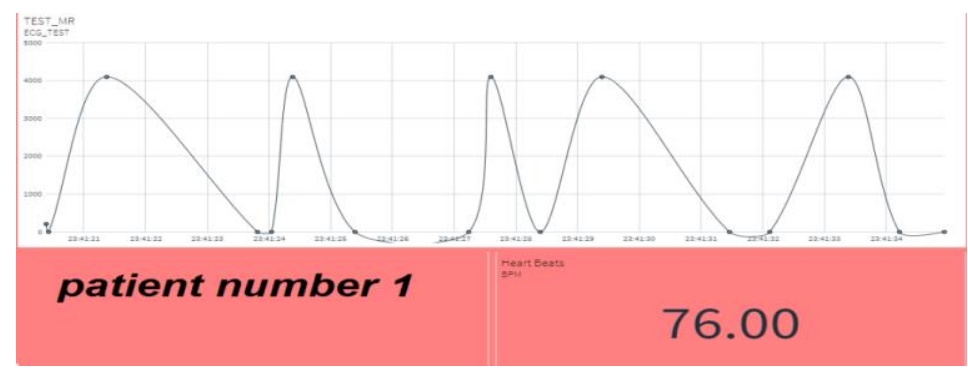

Figure 12. First patient with heart beat

\section{CONCLUSION}

Designed and implemented a system based on advanced Internet technologies for an ECG monitoring. The ECG System construction was initially introduced. The ECG sensor is displayed and compared with different way of transport, including Wi-Fi, Bluetooth, and Zigbee networks. Based on the proposed construction, Through ECG node with three electrodes that can be worn, ECG signals can be collected real-time and transport to anywhere in world. The collected ECG signal was sent to the Internet of things by $\mathrm{Wi}-\mathrm{Fi}$, which uploading data for the wanted coverage areas. It is an independent tool for the patient and is a very useful tool for the doctor using the two methods presented in the paper to know the heart rate, which shows whether the patient has any increase or decrease in heart rate and can take this system with him anywhere with the availability of Wi-Fi through An operating system for browser users and access to ECG data, provides a web-based graphical user interface. In the future, studies need further development of ECG monitoring. Such as the need to improve the accuracy of the ECG signal and provide sensors that will help more patient observation. This will help in the long term with the easiest and least expensive way to present problems in healthcare.

\section{REFERENCES}

[1] N. Athilah, A. Rahman, and A. B. Jambek, "Biomedical health monitoring system design and analysis", vol. 13, no. 3, pp. 1056-1064, 2019.

[2] H. Burri and D. Senouf, "Remote monitoring and follow-up of pacemakers and implantable cardioverter defibrillators", pp. 701-709, 2009.

[3] A. Lazarus, B. Guy-moyat, P. Mondoly, C. Quaglia, J. Elkaim, and S. Bayle, "Active periodic electrograms in remote monitoring of pacemaker recipients : the PREMS study", no. July, pp. 1-7, 2018.

[4] J. L. Anderson et al., "in Patients with a Pacemaker or Defibrillator", pp. 755-764, 2017.

[5] N. Azaliah, A. Bakar, W. Makhtariah, W. Ramli, and N. H. Hassan, "The internet of things in healthcare: anoverview, challenges and model plan for security risks management process", vol. 15, no. 1, pp. 414-420, 2019.

[6] M. S. Uddin, J. B. Alam, and S. Banu, "Real time patient monitoring system based on Internet of Things", 4th International Conference on Advances in Electrical Engineering, ICAEE 2017, vol. 2018-Janua, pp. 516-521, 2018.

[7] Z. M. Yusof, M. Billah, and K. Kadir, "Real-time water quality monitoring system : an IoT application", vol. 15, no. 1, pp. 178-182, 2019.

[8] Z. Yang, Q. Zhou, L. Lei, K. Zheng, and W. Xiang, "An IoT-cloud Based Wearable ECG Monitoring System for Smart Healthcare", Journal of Medical Systems, vol. 40, no. 12, 2016.

[9] C. Doukas and I. Maglogiannis, "Bringing IoT and cloud computing towards pervasive healthcare", Proceedings 6th International Conference on Innovative Mobile and Internet Services in Ubiquitous Computing, IMIS 2012, pp. 922-926, 2012. 
[10] E. Likotiko, D. Petrov, J. Mwangoka, U. Hilleringmann, I. C. Science, and S. Technology, "Real Time Solid Waste Monitoring using Cloud and Sensors Technologies", vol. 8, no. 1, pp. 106-116, 2018.

[11] M. Kohvakka, J. Suhonen, M. Kuorilehto, V. Kaseva, M. Hännikäinen, and T. D. Hämäläinen, "Energy-efficient neighbor discovery protocol for mobile wireless sensor networks", Ad Hoc Networks, vol. 7, no. 1, pp. 24-41, 2009.

[12] M. A. Patricio, "Thinger. io: An Open Source Platform for Deploying", Department of Electronic Engineering, Pukyong National University, no. 1044, 2019.

[13] K. A., K. R., and M. O. http://orcid. org/000.-0001-6578-9741 Kumar M. AO-Kumar, "From Pacemaker to Wearable: Techniques for ECG Detection Systems", Journal of Medical Systems, vol. 42, no. 2, p. 34, 2018.

[14] C. De Capua, A. Meduri, and R. Morello, "A smart ECG measurement system based on web-service-oriented architecture for telemedicine applications", IEEE Transactions on Instrumentation and Measurement, vol. 59, no. 10, pp. 2530-2538, 2010.

[15] Ecg Sensor, "Modren Electroincs", 2017. [Online]. Available: https://modernelectronics.com.pk/product/moduleecg/. [Accessed: 01-Jul-2019].

[16] I. Allafi and T. Iqbal, "Design and implementation of a low cost web server using ESP32 for real-time photovoltaic system monitoring", 2017 IEEE Electrical Power and Energy Conference, EPEC 2017, vol. 2017-Octob, pp. 1-5, 2018.

[17] A. Maier, A. Sharp, and Y. Vagapov, "Comparative analysis and practical implementation of the ESP32 microcontroller module for the internet of things", 2017 Internet Technologies and Applications, ITA 2017Proceedings of the 7th International Conference, pp. 143-148, 2017.

[18] R. K. Kodali and B. S. Sarjerao, "MQTT based Air Quality Monitoring", pp. 21-23, 2017.

[19] B. Lee, J. Kim, H. Kim, C. Kim, and S. Lee, "Low-cost flexible pressure sensor based on dielectric elastomer film with micro-pores", Sensors \& Actuators: A. Physical, 2016.

[20] A. N. A. Journal, "Synthesis and Exploration of Ladder-Structured Large Aromatic Dianhydrides as Organic Cathodes for Rechargeable Lithium Batteries".

[21] O. Faust, "Heart-rate based sleep apnea detection using arduino", vol. 19, no. 1, pp. 1-29, 2019.

[22] "Pacemaker Categorios", ekg.acadmy, 2019. [Online]. Available: https://ekg.academy/pacemaker-rhythms. [Accessed: 10-Aug-2019].

[23] I. C. Follow-up, "Arrhythmia/Electrophysiology", 2010.

[24] J. Mohammed, C. H. Lung, A. Ocneanu, A. Thakral, C. Jones, and A. Adler, "Internet of things: Remote patient monitoring using web services and cloud computing", Proceedings-2014 IEEE International Conference on Internet of Things, iThings 2014, 2014 IEEE International Conference on Green Computing and Communications, GreenCom 2014 and 2014 IEEE International Conference on Cyber-Physical-Social Computing, CPS 20, no. iThings, pp. 256-263, 2014.

[25] R. B. Ambulatory, "Wearable ECG Terminal and Its Telemedicine-Health Services", pp. 133-137, 2011.

[26] J. Welch, F. Guilak, and S. D. Baker, "A Wireless ECG Smart Sensor for Broad Application in Life Threatening Event Detection", pp. 3447-3449, 2005.

\section{BIOGRAPHIES OF AUTHORS}
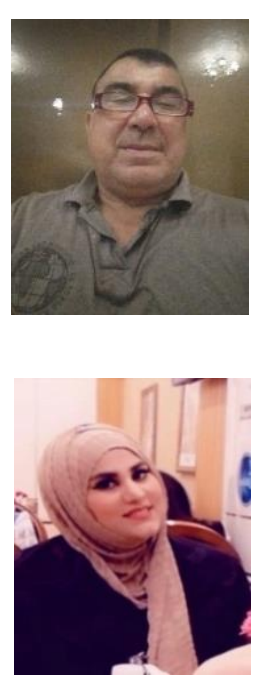

Jameel Kadhim Abed received his MSc and PhD degree in power system, machine and power electronics from the University of Baghdad in 1985 and 2003 respectively. He has worked at the Foundation of Military Production since 1988 as Head Master of the Development and Research Department in the field of power electronics (power supply and inverters). Currently he works in the Middle Technical University as Head Master of the Mechatronics Department and as lecturer in power electronics for undergraduate and postgraduate students as well as supervisor of master and high diploma students in the field of high frequency inverter and renewable energy. His research is on electrical drives and power electronics on various inverter types, and he is interested in renewable energy.

Hassnaa Mustafa Abed is a student of MSC of The Department of Medical Instrumentation Techniques Engineering, Electrical Engineering Technical College 